\title{
Measurements and analysis of a high-brightness electron beam collimated in a magnetic bunch compressor
}

\author{
F. Zhou, K. Bane, Y. Ding, Z. Huang, H. Loos, and T. Raubenheimer \\ SLAC National Accelerator Laboratory, 2575 Sand Hill Road, Menlo Park, California 94025, USA
}

(Received 5 March 2015; published 18 May 2015)

\begin{abstract}
A collimator located in a magnetic bunch compressor of a linear accelerator driven x-ray free electron laser has many potential applications, such as the removal of horns in the current distribution, the generation of ultrashort beams, and as a diagnostic of the beam slice emittance. Collective effects, however, are a major concern in applying the technique. Systematic measurements of emittance and analysis were performed using a collimator in the first bunch compressor of the Linac Coherent Light Source (LCLS). In the nominal, undercompressed configuration using the collimator we find that the $y$ emittance (nonbending plane) is not increased, and the $x$ emittance (in the bending plane) is increased by about $25 \%$, in comparison to the injector emittance. From the analysis we conclude that the parasitic effects associated with this method are dominated by coherent synchrotron radiation (CSR), which causes a "systematic error" for measuring slice emittance at the bending plane using the collimation method. In general, we find good agreement between the measurements and simulations including CSR. However, for overcompressed beams at smaller collimator gaps, an extra emittance increase is found that does not agree with 1D simulations and is not understood.
\end{abstract}

DOI: 10.1103/PhysRevSTAB.18.050702

PACS numbers: 29.27.-a

\section{INTRODUCTION}

Over the past several years, great progress has been made in the realization of high power $\mathrm{x}$-ray free-electron lasers (FELs) [1-2]. The typical x-ray FEL has $10^{11}-10^{13}$ photons per pulse, a pulse power of 10's-100's of gigawatts, and a pulse duration from a few to several hundred femtoseconds. These new machines have become revolutionary tools for ultrafast, time-resolved studies of atomic and molecular worlds. However, there is a continual demand for the development of new techniques to reach ever higher power-to the terawatt scale, and ever shorter pulse lengths - to the subfemtosecond scale. To drive an x-ray FEL, an electron bunch needs to be accelerated to high energies and compressed to a high peak current [1]. The compression is normally achieved by first inducing correlated energy spread along the bunch in time (an energy chirp) and then passing it through a magnetic chicane; and this process is often repeated at least once during beam acceleration. However, nonlinear terms in the chirp, caused by accelerator structure wakefields [3-6], typically result in a final bunch shape that is double horned-with one horn leading and the other trailing-rather than one that is uniform [7]. In the horn regions of the distribution the peak current can be greatly enhanced (compared to the current in

\footnotetext{
*Zhoufeng@slac.stanford.edu

Published by the American Physical Society under the terms of the Creative Commons Attribution 3.0 License. Further distribution of this work must maintain attribution to the author $(s)$ and the published article's title, journal citation, and DOI.
}

the core), resulting in negative consequences such as: in the chicane, induced coherent synchrotron radiation (CSR) [8-10] leading to emittance growth $[9,11]$, and a limit on the bunch compression that can be achieved; in the undulator, an increase in wake-induced energy variation and a nonuniformity in the lasing process [12]. Also, having a nonuniform beam distribution in the undulator makes it difficult to transversely match the beam. Indeed, the horns can significantly degrade the FEL performance [7]. Removing particles from the distribution so that there are no horns can yield an improved FEL performance, even though there are then fewer particles that reach the undulator.

Recently, a technique - where a collimator in the middle of a chicane is used to slice the beam-was experimentally investigated for the purpose of beam diagnostics at Fermi FEL [13]. At about the same time, the same technique was employed at the Linac Coherent Light Source (LCLS) [14] to generate a distribution without horns in order to improve FEL performance [15]. In the LCLS there are two fourdipole chicanes (called $\mathrm{BC} 1$ and $\mathrm{BC} 2$ ) and the collimator located in the middle of the first one (BC1) is used for these studies (because the energy is lower and thus less background radiation is produced). In the center of a chicane the chirped beam is tilted in the longitudinal-horizontal $(z-x)$ plane, and collimating in $x$ cuts away head and tail particles in the bunch distribution. At the LCLS it was found that, with proper adjustment, a relatively uniform final temporal distribution can be obtained. In Fig. 1 we show simulation examples of the LCLS beam at the entrance of the undulator, comparing the results of the 

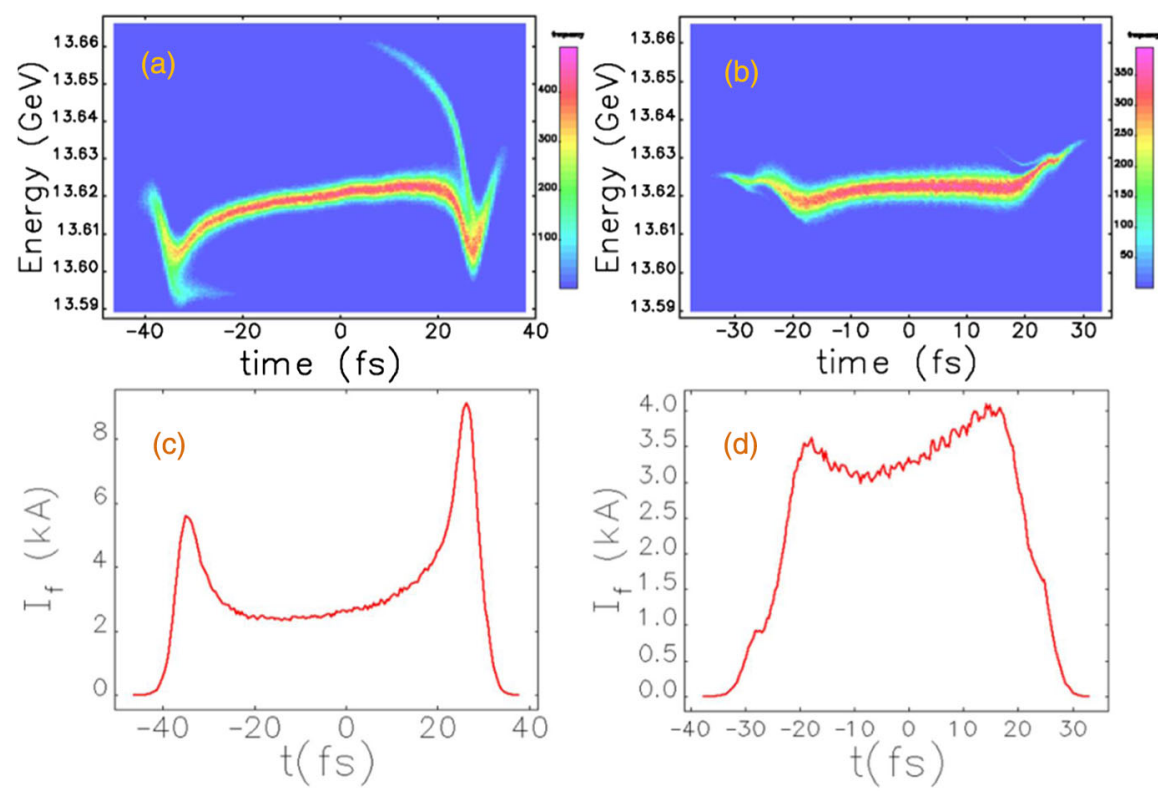

FIG. 1. Simulated electron beam phase space and current profile at the entrance of the LCLS undulator. (a) and (c) are for regular compression setup with full charge of $250 \mathrm{pC}$ without collimation; (b) and (d) show the collimated beam cutting down to about $150 \mathrm{pC}$ using the $\mathrm{BC} 1$ collimator. We see clearly the reduced current horns and flat phase space with the truncation mode.

normal configuration [(a) and (c)] with that of the collimated beam [(b) and (d)]. In this example, the beam begins at the injector with $250 \mathrm{pC}$ of charge; head and tail particles are removed with a collimator in $\mathrm{BC} 1$, so that the charge remaining becomes $150 \mathrm{pC}$. With the truncated current profile after $\mathrm{BC} 1$, we find through simulations that we are able to produce a relatively uniform final beam distribution with small horns. The projected emittance of the truncated beam at the undulator entrance is about a factor 2 smaller than for the regular double-horned beam distribution at a similar current. The beam power loss at the collimator is small, only 1-2 Watts when the $250 \mathrm{pC}$ beam is collimated down to 200 or $150 \mathrm{pC}$ at $\mathrm{BC} 1(220 \mathrm{MeV}, 120 \mathrm{~Hz})$. Recently the above-described horn collimation has been used in LCLS operations.

In the absence of horn particles the bunch can be compressed more strongly in $\mathrm{BC} 2$, thereby increasing the flexibility in running the FEL [15]. In addition, when used in self-seeding mode [16]—-where a narrow energy distribution in the undulator is especially important-horn collimation has been shown to be of great help in approaching a Fourier-transform limited bandwidth. Thus, this method with the collimator set to a reasonably wide aperture (about $1 / 2$ of the fully dispersed beam size), can be used to avoid horns in the final bunch distribution. This same idea, but with the collimator set to a very narrow aperture (about $1 / 10$ or $1 / 20$ of the fully dispersed beam size), so that only a small fraction of the beam passes through, can also be used to create very short x-ray pulses (as was also suggested in [13]), and as a diagnostic to measure the time-sliced emittance of the beam $[13,14]$.
This is a simple and inexpensive method of shaping the beam current distribution, of generating an ultrashort beam, and of measuring the time-resolved emittance.

We see that a collimator in the middle of a chicane has several potential applications. One important question that needs to be addressed, however, is how important are the collective effects when collimating a beam in this way? For example, can the collimation increase the emittance? Or, when collimating strongly to perform a time-sliced emittance measurement, how do the excited collective effects affect the results and does the measured emittance of a collimated beam represent a true slice emittance? This report describes measurements at the LCLS and analysis of the collimator method described above. Compared to the work in Ref. [13], the present paper focuses more on systematic measurements and analysis of the role of the collective effects on the measured results, and points out that the measured emittance of the physically collimated slice beam is larger than true slice emittance due to the CSR effect in the bending plane. The paper is organized as follows. In Sec. II, the LCLS injector emittance is presented, serving as a reference for the subsequent collimation studies. Section III describes the implementation of the collimation scheme at LCLS BC1 and presents measured data and analysis. The results are summarized in Sec. IV. Note that in this paper we use the term "slice emittance" to refer to the emittance of a small (longitudinal) slice of the beam. If different slice beam phase space has correlation (or misalignment), the "projected emittance" of the whole beam will increase, but the slice emittance will remain unaffected. 


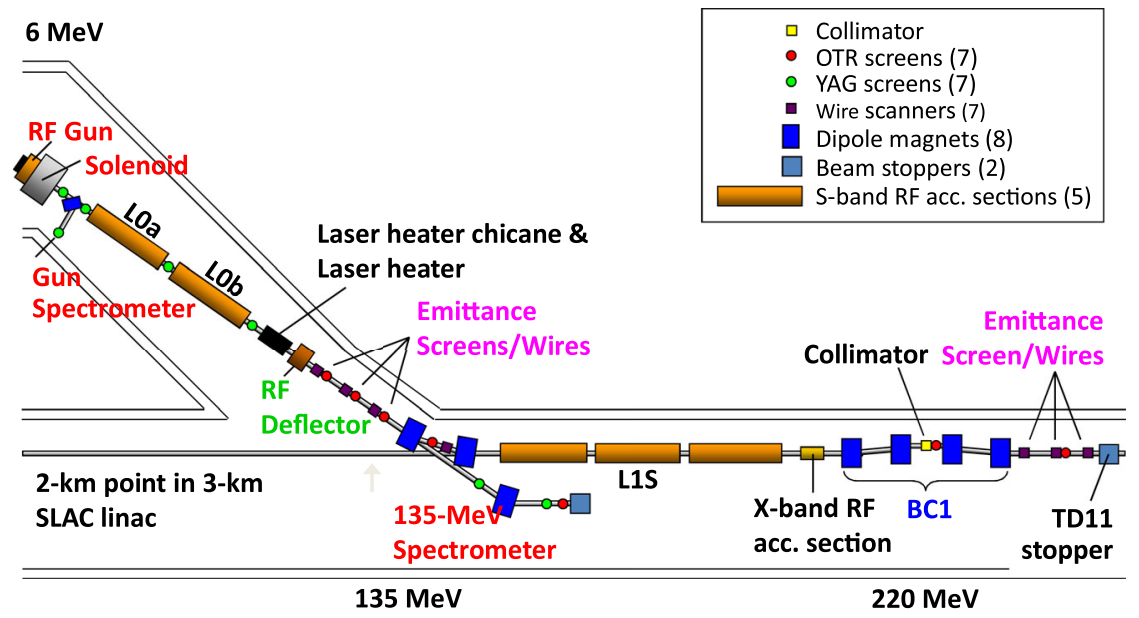

FIG. 2. Schematic layout of the LCLS injector and BC1. The horizontal collimator is located in the center of the BC1 chicane.

\section{BEAM MEASUREMENTS AT THE LCLS INJECTOR}

Figure 2 presents a schematic of the LCLS injector and the first stage of magnetic bunch compressor $\mathrm{BC} 1$ regions. It begins with an $S$-band radio frequency (rf) gun. This is followed by two $S$-band linac sections, where the beam is accelerated to an energy of $135 \mathrm{MeV}$ [17]. The beam then passes through a small laser heater (LH) chicane [18]; then through an $S$-band, transverse rf deflector, which can be used for time-resolved, beam property measurements. Then follow three optical transition radiation (OTR) screens and several wire scanners; these diagnostics can be used to measure emittance. For easy descriptions in the subsequent sections, we call the emittance measured at this point as the $135-\mathrm{MeV}$ injector emittance. The beam then passes through the $S$-band accelerating section, L1S, where it is accelerated to $240 \mathrm{MeV}$; and then through the $X$-band linearizer, L1X [17]. Then follows the first (four-bend) chicane, BC1, where the beam is longitudinally compressed. Note that it is a horizontal collimator located at the center of this chicane that we use in our studies. Finally, downstream of the chicane are wire scanners that can be used to measure the emittance of the compressed bunch.

To evaluate the effect of the collimator on the emittance, we first need to accurately characterize the incoming injector emittance upstream of the collimator. The $135-\mathrm{MeV}$ injector emittance used to be measured using an OTR screen with the LH chicane on, but then it was found that the emittance measured this way was often in error and underestimated. This is because the LH chicane can induce coherent optical transition radiation (COTR) [19], an effect that tends to make core beam brighter thereby resulting in smaller beam size. Figure 3 compares, for charge $Q=150 \mathrm{pC}$, the (normalized) horizontal emittance measured by the OTR screens and by the wire scanners (used in combination with quad scans), for cases LH chicane on and off. The wire scanner measurements are not affected by COTR, and we see that the emittance measured using them (for both LH chicane on and off) is similar to the one obtained using the OTR screen with the LH chicane off. The measured $x$ - and $y$-emittance is very close, i.e., $\varepsilon_{x n} \approx \varepsilon_{y n} \sim 0.45 \mu \mathrm{m}$. These results indicate that the (projected) emittance measurement is reliable either when using the wire scanners or when using the OTR screen with the LH chicane turned off.

Slice emittance is an important beam parameter in an FEL machine. The natural length scale along the bunch is the slippage length, defined as the longitudinal slippage of the electrons with respect to the photons over the undulator length. For x-ray FELs, this slippage length is typically a small fraction (called a "slice") of the electron bunch. So this slice emittance is a time-resolved emittance along a long bunch, and it is typically smaller than the projected

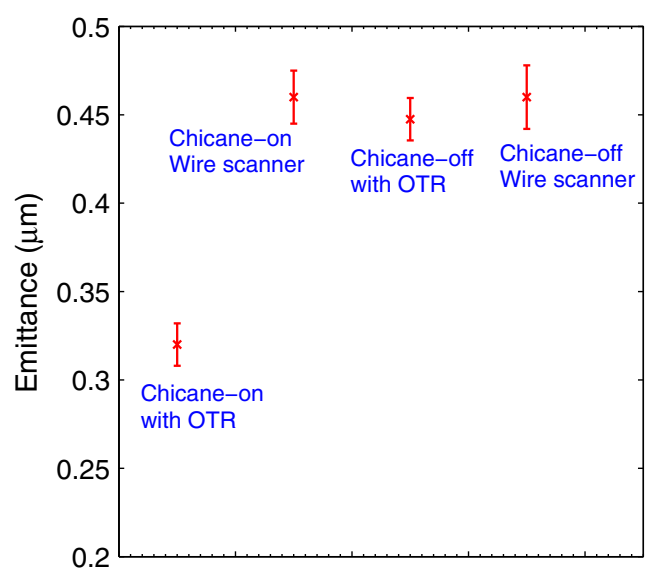

FIG. 3. Projected emittance measured at the $135 \mathrm{MeV}$ point of the LCLS injector using the OTR screen and the wire scanner, for cases LH chicane on and off. The measured emittance $\varepsilon_{x n}$ is very close to $\varepsilon_{y n}$. The beam charge is $150 \mathrm{pC}$ and the peak current is about $50 \mathrm{~A}$ for the measurements. 


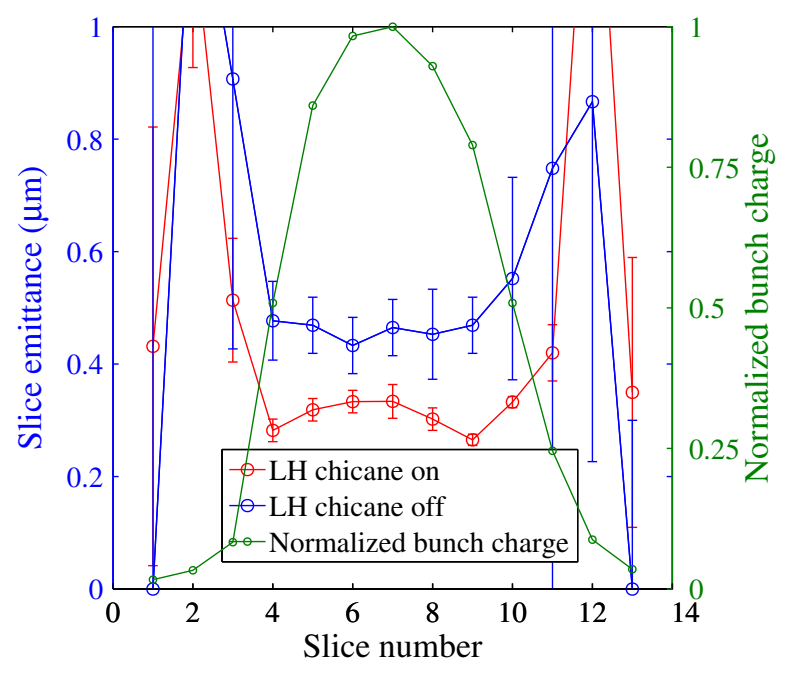

FIG. 4. Horizontal slice emittance, comparing the cases LH chicane on and off, as measured in the LCLS injector at $135 \mathrm{MeV}$, for $Q=150 \mathrm{pC}$. The bunch shape is given in green. Note that emittance values measured in the beam tails are inaccurate and should be ignored.

emittance of the whole bunch especially after a bunch compression [20]. The slice $x$-emittance inside a long bunch can be measured using the rf deflector [17] in combination with a downstream OTR screen. Results are given in Fig. 4, where we show slice emittance with the LH chicane off (blue) or on (red); the bunch shape is given in green. We see that, over the beam core, the measured slice $x$-emittance at $135-\mathrm{MeV}$ injector with the LH chicane on is again smaller than when it is off. Note the emittance measurements in the beam tails are inaccurate and should be ignored. We conclude that the normalized slice $x$-emittance (in the beam core) at the $135-\mathrm{MeV}$ injector is the value obtained with the LH chicane off: $\varepsilon_{x n} \sim$ $0.45 \mu \mathrm{m}$ for $Q=150 \mathrm{pC}$. Since the projected $x$ - and $y$-emittance is very close, it is reasonable to assume the measured slice $y$-emittance is similar to slice $x$-emittance, $0.45 \mu \mathrm{m}$ for $Q=150 \mathrm{pC}$. We take this as the reference emittance for the following collimator studies. Note all measurements shown in Figs. 3 and 4 have $0.4 \mathrm{~mm}$ of rms bunch length of full beam (i.e., about $50 \mathrm{~A}$ of peak current) and the full beam is equally sliced into 13 pieces for slice emittance measurements (i.e., each slice has about $30 \mu \mathrm{m}$ in length).

\section{MEASUREMENT AND ANALYSIS FOR A COLLIMATED BEAM}

The collimator located at the LCLS BC1 center is used for the beam collimation studies. A sketch of the layout of the LCLS BC1 is shown in Fig. 5. The BC1 chicane contains four horizontal bending magnets. A flat, horizontal collimator is located at the center of the chicane, where the designed $x$-dispersion is $\eta_{x}=-0.23 \mathrm{~m}$ under nominal operation. Just downstream of the collimator is an OTR screen, one that can be used for imaging the electron beam profile. In the upstream linacs, L1S and L1X, the electron beam rides ahead of the rf crest, resulting in a positive timeenergy chirp (with the bunch head at lower energy than the tail). The bunch $x-t$ tilt reaches a maximum at the center of the chicane. The rms dispersed beam size is $\sigma_{x}=2.3 \mathrm{~mm}$ (much larger than betatron beam size, $\sigma_{x \beta}=90 \mu \mathrm{m}$ ), and the correlated energy spread is $\sigma_{\delta}=1 \% \mathrm{rms}$. The full beam size at the BC1 OTR screen is $\sim 11 \mathrm{~mm}$ with $150 \mathrm{pC}$. Major parameters for the $\mathrm{BC} 1$ chicane are given in Table I. The peak current is increased to $220 \mathrm{~A}$ through the $\mathrm{BC} 1$ bunch compression.

In beam collimation mode, the collimator gap is narrowed to scrape away electrons. As discussed earlier, due to the $x$ - $t$ tilt in the bunch at the location of the collimator, electrons at the head and tail of the bunch can be removed by closing the collimator jaws. Figure 6 (bottom) shows two examples of truncated profiles measured at the OTR screen. At the LCLS, to avoid beam horns with initial charge $Q=150 \mathrm{pC}$, we close the collimator gap to $g=5 \mathrm{~mm}$ and end up removing $\sim 1 / 3$ of the charge. For the applications of generating ultrashort pulses or measuring the slice emittance of a beam, the collimator gap is set to a smaller value. The minimum gap is usually 3-5 times $\sigma_{x \beta}, 0.3-0.5 \mathrm{~mm}$, so that the dispersed beam size can dominate. The emittance of the short, collimated beam can be measured with the wire scanner just downstream of $\mathrm{BC} 1$.

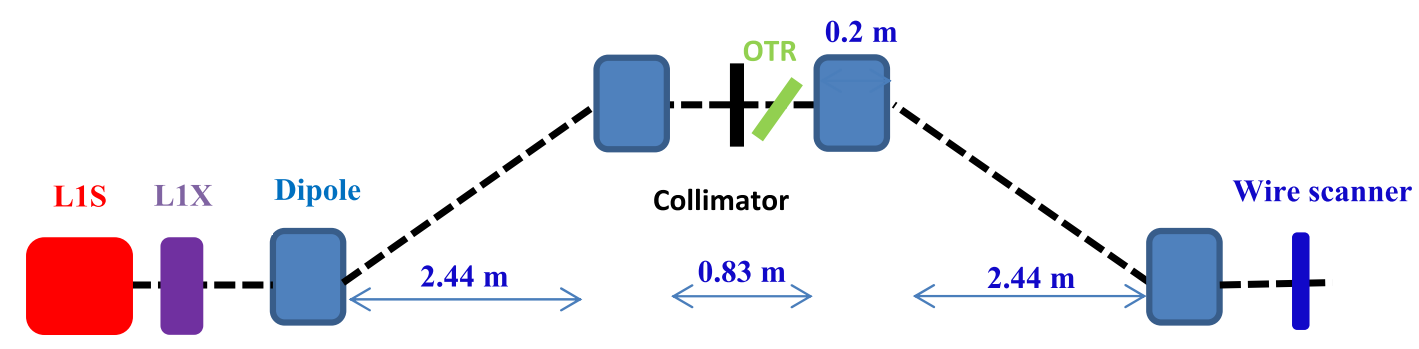

FIG. 5. Sketch of the LCLS first chicane, BC1 (not scaled). The linac L1S is used to introduce an energy-time correlation needed for bunch compression at $\mathrm{BC} 1$, while the $x$-band linac L1X is used to linearize longitudinal phase space. The wire scanner for emittance measurements is located just downstream of $\mathrm{BC} 1$. Each bend length is $0.2 \mathrm{~m}$ and bending radius is $2 \mathrm{~m}$. 
TABLE I. Twiss and beam parameters at the LCLS BC1 chicane center.

\begin{tabular}{lcc}
\hline \hline Parameter & Value & Unit \\
\hline Energy, $E$ & 220 & $\mathrm{MeV}$ \\
Twiss parameters, $\beta_{x} / \beta_{y}$ & 7 & $\mathrm{~m}$ \\
Betatron beam size $x / y$ & 0.09 & $\mathrm{~mm}$ \\
$x$-dispersion & -0.23 & $\mathrm{~m}$ \\
rms energy spread & $1 \%$ & $\ldots$ \\
rms dispersive beam size & 2.3 & $\mathrm{~mm}$ \\
\hline \hline
\end{tabular}

\section{A. Emittance measurement for a collimated beam}

Under normal operating conditions, the L1S phase is set to between $\varphi=-26^{\circ}$ and $-27^{\circ}$ (ahead of crest) for an undercompressed beam, which yields a peak current of $I=220$ A after BC1. In this configuration, for a bunch charge $Q=150 \mathrm{pC}$, the projected emittance and transmitted charge were measured as functions of collimator gap, $g$ (see Fig. 7). We see that the emittances remain relatively unchanged, whether the gap is fully open $(g=10 \mathrm{~mm})$, or closed down to $g=1 \mathrm{~mm}$; and that the vertical emittance is the same as in the injector $\varepsilon_{n y}=0.45 \mu \mathrm{m}$, but the horizontal emittance has grown by $25 \%$ in comparison to the injector emittance, to $\varepsilon_{n x}=0.55 \mu \mathrm{m}$. The later analyses show that the CSR is responsible for the emittance growth for the collimated slice beam. The details are described in Sec. III B.

Next, with a small gap of $g=0.5 \mathrm{~mm}$, by varying its location in $x$, the emittance of different time slices of the bunch was measured. In this configuration, out of an initial total charge $Q=150 \mathrm{pC}$, about $10 \mathrm{pC}$ is allowed to pass through the collimator jaws. After a second stage compression the collimated beam with $10 \mathrm{pC}$ is only a few femtoseconds which is close to an FEL slippage length. The measurement results are given in Fig. 8. There is some

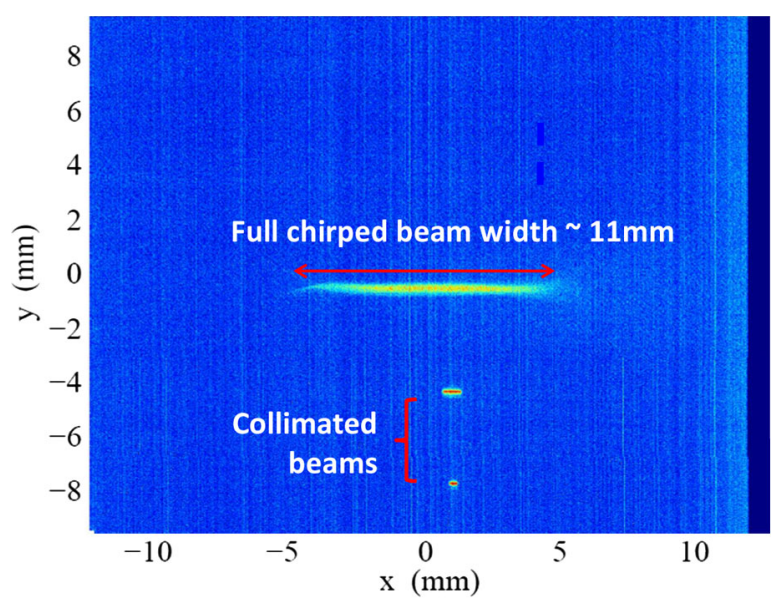

FIG. 6. Examples of measured beam images at the OTR screen just downstream of the collimator: the full, $150 \mathrm{pC}$ chirped beam (top) and collimated beams with different collimator gaps (middle and bottom).

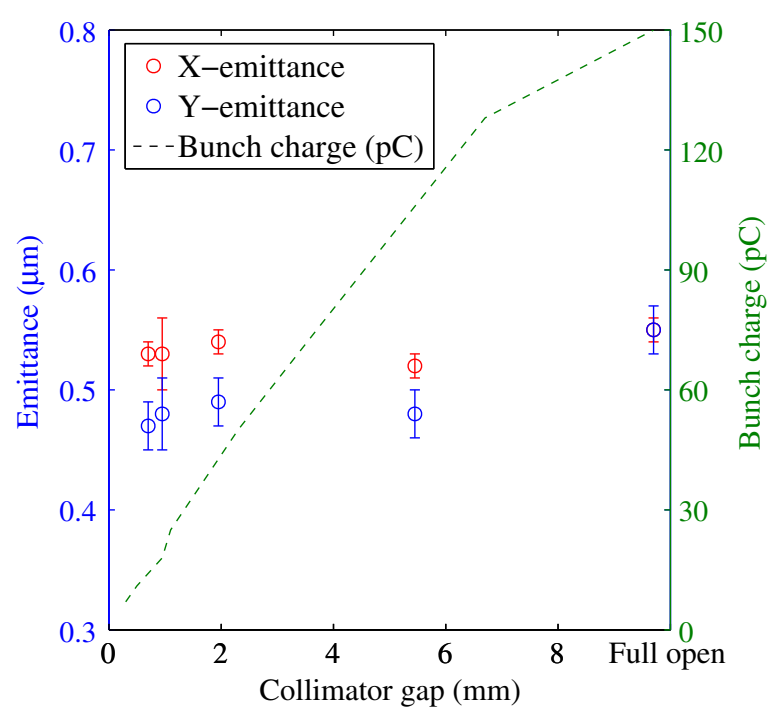

FIG. 7. Measured $x / y$-emittances and charge vs collimator gap. The collimator gap $g=10 \mathrm{~cm}$ for the full open case.

scatter in the results (results in the tails of the bunch distribution are less accurate) but we see that the measured $y$-emittance of the strongly collimated beam is about $0.45 \mu \mathrm{m}$ (similar to the sliced emittance at $135-\mathrm{MeV}$ injector, before the $\mathrm{BC} 1$ ), which is smaller than the one without the collimation. The correlation (or misalignments) of slice beam phase spaces particularly for head and tail slices are generated in the $x$-band linearizer upstream of the $\mathrm{BC} 1$ due to its short-range wake. The correlation of head and tail slices can be removed with this strong collimation. The measured core $x$-emittance is notably larger, $\varepsilon_{n x} \sim 0.55-0.60 \mu \mathrm{m}$, having grown $25 \%-30 \%$ from the injector. Note again according to the slice emittance concept, it is a longitudinally resolved emittance along a

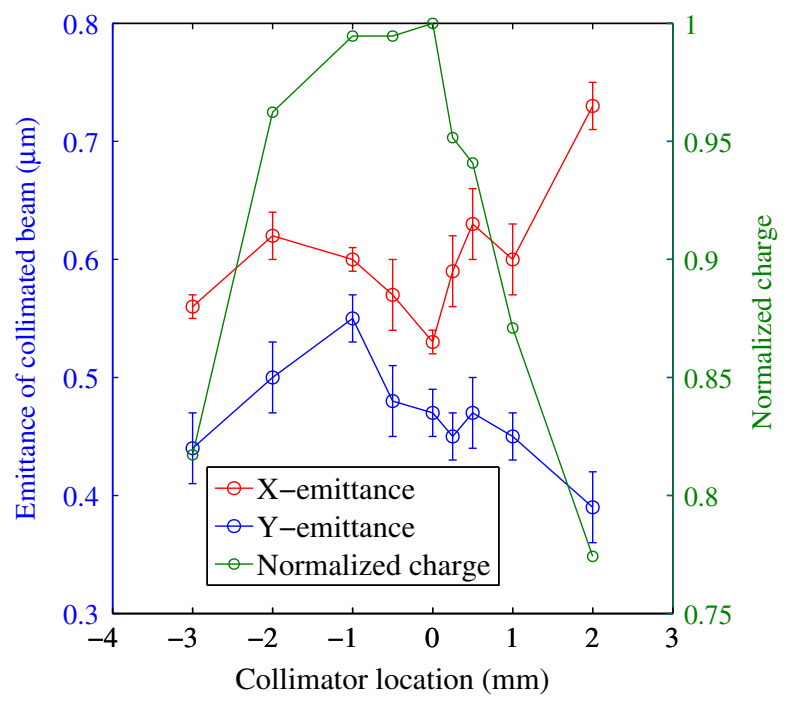

FIG. 8. Measured $x$ - and $y$-emittance of collimated slice beam along the long bunch with $g=0.5 \mathrm{~mm}$ of collimator gap. 

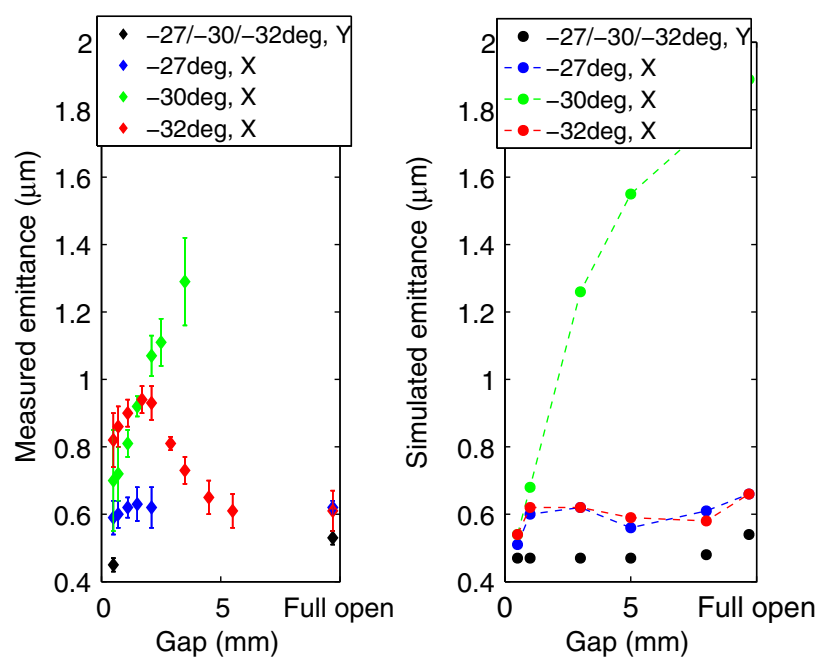

FIG. 9. Measured (left) and simulated (right) emittance vs collimator gap for different amounts of bunch compression. The L1S phase of $-27^{\circ},-30^{\circ}$ and $-32^{\circ}$ corresponds to under compression, full compression and overcompression, respectively.

bunch. However, here we measure projected emittance of a physically truncated slice beam through cutting from a long bunch to an isolated short slice beam.

Next we performed systematic emittance measurements, for under compressed, over compressed, and fully compressed configurations, as functions of collimator gap. In Fig. 9 (left), we present the results for cases of (near nominal) undercompression (linac L1S phase $\varphi=-27^{\circ}$ ), fullcompression $\left(\varphi=-30^{\circ}\right)$, and overcompression $\left(\varphi=-32^{\circ}\right)$. For all three cases and all gaps we find that the final measured $y$-emittance remained unchanged and close to the initial value, $\varepsilon_{y n}=0.45 \mu \mathrm{m}$. The measured $x$-emittance, however, always increased in comparison to the injector emittance. At undercompression, we obtained $\varepsilon_{x n}=$ $0.60 \mu \mathrm{m}$ for all gaps. Note that these results are slightly larger than those of the earlier measurements shown in Fig. 7; we believe this is likely due to day-to-day variation in the initial beam properties and/or the machine configuration. At full compression, at full gap we obtained $\varepsilon_{x n}=1.8 \mu \mathrm{m}$; as the gap was reduced, the measured emittance decreased monotonically down to $\varepsilon_{x n}=0.7 \mu \mathrm{m}$ at the smallest gap. At overcompression, we obtained $\varepsilon_{x n}=0.60 \mu \mathrm{m}$ at the larger gaps; as the gap was decreased, however, a large bump in the data appeared, one that reached to $\varepsilon_{x n}=0.93 \mu \mathrm{m}$ at a gap $g=1.5 \mathrm{~mm}$. The bump in the data was repeatable as it was found again in data taken on another day. In the following section we attempt to make sense of these results by simulations including the effect of coherent synchrotron radiation $(\mathrm{CSR})$.

\section{B. Coherent synchrotron radiation}

Short electron bunches traversing a dipole can emit CSR at wavelengths longer than the bunch length. The effects can be described as a wakefield effect in that it can increase the projected emittance. The CSR wake, in general, is a 3D effect that can require quite complicated computer algorithms for its calculation. Often, however, a much simpler 1D steady state or slightly more complicated 1D transient formula [21] — where the latter includes the effects of the magnet ends in a series of bending magnets, as is found in a chicane-gives reasonably accurate results. Note that when the code ElEgant [22] calculates the CSR effect, it employs the latter, transient formula [23].

In a four-dipole chicane compressor, like $\mathrm{BC} 1$ in the LCLS, the CSR effect is normally strongest in the last one or two bends, where the bunch is the shortest. The CSR caused $x$-emittance growth for a collimated beam (we assume the bending is in $x$-plane) can be estimated with the 1D steady-state model [24]:

$$
\varepsilon_{x n}=\varepsilon_{x n 0} \sqrt{1+\left(\frac{\theta}{2} \frac{\delta_{\mathrm{CSR}}}{\sigma_{x 0^{\prime}}}\right)^{2}},
$$

where $\varepsilon_{x n}\left(\varepsilon_{x n 0}\right)$ are the final (initial) emittance, $\theta$ is the bending angle, $\sigma_{x 0^{\prime}}$ is the original beam divergence; CSR caused rms energy spread, $\delta_{\mathrm{CSR}}$, is given by the unshielded steady state formula [25-26]:

$$
\delta_{\mathrm{CSR}} \approx 0.532 \frac{e Q}{E} \frac{Z_{0} c L}{2 \times 3^{4 / 3} \pi \rho^{2 / 3} \sigma_{z}^{4 / 3}} .
$$

Here $e$ is the charge of the electron, $c$ is the speed of the light, and $Z_{0}=377 \Omega ; Q$ is the bunch charge, $E$ is the beam energy, and $\sigma_{z}$ the rms bunch length; $L$ is the bend length, and $\rho$ the bending radius of the magnet [Note that Eq. (2) assumes that the bunch has a Gaussian longitudinal distribution; however, it can still give a good approximation for other smooth distributions, e.g., a parabolic distribution]. Note that when Eqs. (1) and (2) are applied to a collimated beam in the middle of a chicane, the beam parameters to be used are for that part of the beam that passes through the collimator jaws. During collimation, the charge $Q$ and $\sigma_{z}$ are reduced together, so $\delta_{\mathrm{CSR}} \propto \sigma_{z}{ }^{-1 / 3}$, which is a weak dependence on the bunch length. This can explain why the emittance growth is almost constant as the collimator gap is getting smaller as shown in Fig. 7.

Figure 9 shows the comparisons between measurements (left) and simulations (right) using Elegant code with the transient 1D CSR wake activated. For the simulations, the initial emittance before the LCLS BC1 are $\varepsilon_{n x 0}=\varepsilon_{n y 0}=$ $0.47 \mu \mathrm{m}$. We see in the simulations that the $y$-emittance is not perturbed with different gaps from sub-mm to a few $\mathrm{mm}$ for all three compression scenarios, similar to the incoming emittance, which agrees quite well with the measurements.

From both the measurements and simulations shown in Fig. 9, the $x$-emittance is larger than the $y$-emittance at different gaps for all compression scenarios. If we turn off 
CSR in the simulations, we find that the $x$-emittance is exactly the same as the $y$-emittance for all cases. With the CSR on, the simulated $x$-emittance growth for the undercompressed case $\left(\varphi=-27^{\circ}\right.$ of L1S) and fully compressed case $\left(\varphi=-30^{\circ}\right)$ agree well with the measurements. With the steady-state CSR model Eqs. (1) and (2) give reasonable agreement with the more accurate, transient results of the Elegant code, for both under compressed and fully compressed cases. In the normal operation with the undercompression case $\left(\varphi=-27^{\circ}\right)$, the measured $x$-emittance is very close for the collimator gap $g=5-6 \mathrm{~mm}$ (horn cutting) and $g=0.5 \mathrm{~mm}$ (ultrashort beam generation and/or slice beam diagnostic), which increases about $25 \%$ due to the CSR effect in comparison to the $y$-emittance or incoming slice emittance measured at the $135-\mathrm{MeV}$ injector. In Ref. [13], we noticed their measured $x$-emittance of a collimated slice beam is notably larger than $y$-emittance.

The emittance growth for a collimated beam shown here and also in Ref. [13] could be explained by the CSR effects. As discussed in Ref. [20], during the bunch compression the CSR causes a time-dependent transverse kick and centroid offset. This variation along the bunch is the major reason to cause the projected emittance growth. When we slice the bunch in the time dimension along the bunch, each slice itself has a constant CSR induced kick and offset. So the calculated emittance for an individual slice within a bunch is not affected by the constant kick [20]. Our simulations with Elegant code confirmed that, without the beam collimation, the true time-sliced $x$-emittance after bunch compression is similar to the ones before the bunch compressions. However, for a physically collimated slice beam, it directly experiences the time-dependent CSR effect thereby the measured projected emittance of the collimated slice beam includes the CSR effect. Thus, the collimation technique can be used for slice beam emittance measurements in the nonbending plane but in the bending plane the measured projected emittance of the collimated slice beam is affected by the CSR effect, which is larger than the true slice emittance. For the overcompressed case $\left(\varphi=-32^{\circ}\right)$ for large gap $(g=5-6 \mathrm{~mm}$ for horn cutting application) there is agreement between measurements and simulations. However, for small gaps the anomalous bump found in the measurements is not seen in the simulations with 1D CSR effect. Further investigation with a more complicated 3D CSR model is needed to understand the anomalous bump.

\section{Collimator wakes and dispersion leakage}

In this section we evaluate the collimator wakefield and dispersion leakage effects on the emittance growth. As discussed in the previous section, the typical full width of the chirped beam at the LCLS BC1 chicane center is about $11 \mathrm{~mm}$ when $Q=150 \mathrm{pC}$. During the emittance measurements with different collimator gaps shown in Fig. 6, the beam optics Twiss parameters are not notably affected even at the smallest gaps. This indicates that there is no significant defocusing introduced by the collimator wake. For the case of very short bunches $\sigma_{z} \ll b=g / 2$, valid for our case, the flat collimator quadrupole wake can be predicted using the optical model $[27,28]$. With the beam centered between the collimator jaws, the collimator wake induced beam defocusing is estimated by

$$
\frac{1}{f} \approx \frac{Z_{0} c \pi}{24} \frac{Q}{b^{2}(E / e)}
$$

where $f$ is the effective optical focal length caused by the collimator wake (defocusing is in the plane of the collimation), $Z_{0} \approx 377 \Omega, c$ is the speed of light, $Q$ is the bunch charge transmitted through the collimator, and $E$ is the electron beam energy. The defocusing depends on position within the bunch, with the bunch head experiencing no effect and the tail experiencing the maximum effect, described by Eq. (3). With the LCLS BC1 parameters, beam energy $E=220 \mathrm{MeV}$, the typical transmitted charge $Q=10 \mathrm{pC}$ through the minimum gap of $g=0.5 \mathrm{~mm}$, the focal length created by the collimator wake is $f \sim 90 \mathrm{~m}$, much larger than the few meters of the focal length of the lattice optics. This confirms that the measured Twiss parameters should not be affected even for the smallest collimator gap used. The emittance growth in the $x$ - and $y$-plane due to the collimator's quadrupole wake can be approximately expressed by

$$
\frac{\varepsilon_{n x, n y}}{\varepsilon_{n x 0, n y 0}} \approx \sqrt{1+\left(\frac{\beta_{x, y}}{f}\right)^{2}},
$$

where $\varepsilon_{n x 0, n y 0}$ and $\varepsilon_{n x, n y}$ are the original and final emittances respectively, and $\beta_{x, y}$ is the beta function at the collimator. With nominal $\beta_{x, y}=7 \mathrm{~m}$, the emittance growth caused by the collimator quadrupole wake is $\sim 1 \%$. Further calculations find that the resistive wall quad wake effect of the collimator is even weaker than the collimator's geometrical wake effect, given by Eq. (4). The measurement and analysis lead to the conclusion that the contribution of the collimator wakes to the projected $x$ - and $y$-emittance of a slice beam is negligible.

Dispersion leakage out of the chicane is also a potential source of $x$-emittance growth. The dispersion induced $x$-emittance growth is estimated by [24]

$$
\frac{\Delta \varepsilon_{x}}{\varepsilon_{x 0}} \approx \frac{1}{2} \frac{\left(\eta_{x} \cdot \delta_{E}\right)^{2}}{\beta_{x} \cdot \varepsilon_{x 0}},
$$

where $\Delta \varepsilon_{x}$ is the emittance growth, $\varepsilon_{x 0}$ is the initial emittance, $\beta_{x}$ is the betatron function at the emittance measurement station about $1 \mathrm{~m}, \eta_{x}$ is dispersion and $\delta_{E}$ is the rms energy spread. As only $<7 \%$ of the full beam is selected for one beam slice measurement, the rms energy 
spread of a slice is about $0.07 \%$ for $1 \%$ of the rms energy spread with a full beam. $1 \mathrm{~cm}$ of $x$-dispersion leakage from $\mathrm{BC} 1$ is assumed as a worst case. Substituting the parameters into the Eq. (5) gives negligible $x$-emittance growth for a sliced beam with initial emittance $\varepsilon_{n x}=0.45 \mu \mathrm{m}$.

\section{SUMMARY}

In an x-ray FEL, slicing the beam using a collimator at the center of a chicane bunch compressor is a simple and inexpensive method of shaping the beam current distribution, of generating shorter and higher power pulses, and of measuring the slice beam emittance. The method has been used in the first bunch compressor $\mathrm{BC} 1$ of the LCLS, in order to avoid horns in the final current distribution. Our measurements and analysis in this paper show that most of the parasitic collective effects associated with collimation-such as collimator wakefields, dispersion leakage, and longitudinal space charge-are negligible. Only CSR increases the emittance of a collimated beam in the bending plane. At the nominal undercompressed setting of $\mathrm{BC} 1$, in comparison to the incoming emittance the emittance growth is about $25 \%$, a value that does not depend on the collimator gap. The collimator technique can be used for removing the beam horns, as the machine operation becomes more flexible and the FEL performance is improved.

Used as a narrow slicing tool, our measurements show that the results are reliable in the nonbending plane, close to the measured slice emittance at the injector upstream of the $\mathrm{BC} 1$; in the bending plane, however, the measured projected emittance of the collimated slice beam becomes larger than the true slice emittance of the nonphysically collimated beam due to the CSR effect. Thus, the narrowed collimator is a reliable diagnostic for the slice emittance of the beam in the nonbending plane but not in bending plane. In this paper, we also compared the measurement results to simulations using Elegant with its 1D model of CSR activated, for cases of under compression, full compression, and overcompression, and found reasonable agreement. For the overcompression case with smaller gaps, however, we find an anomalous growth in emittance that does not agree with 1D simulation and is not yet understood.

Although the measurements were done at the LCLS, this collimation scheme can be applied to other FEL facilities operating with normal conducting linac. This provides a simple method for x-ray pulse shaping and FEL performance improvement, and slice emittance measurements in the nonbending plane.

\section{ACKNOWLEDGMENTS}

The work is supported by U.S. DOE Contract No. DEAC02-76SF00515. We would like to thank LCLS physicists for the fruitful discussions.
[1] P. Emma et al., First lasing and operation of an Angstromwavelength free electron laser, Nat. Photonics 4, 641 (2010).

[2] T. Ishikawa et al., A compact x-ray free-electron laser emitting in the sub-angstrom region, Nat. Photonics 6, 540 (2012).

[3] S. Di Mitri and M. Cornacchia, Phys. Rep. 539, 1 (2014).

[4] K. L. F. Bane, Report No. SLAC-PUB-11829, 2006.

[5] S. Di Mitri et al., in Proceedings of the 27th International Free Electron laser Conference, Stanford, USA, 2005, pp. 130-133.

[6] T. O. Raubenhemier, Estimates of emittance dilution and stability in high-energy linear accelerators, Phys. Rev. ST Accel. Beams 3, 121002 (2000).

[7] Linac Coherent Light Source (LCLS) Conceptual Design Report, SLAC Report No. SLAC-R-593, 2002.

[8] Ya. Derbenev et al., Report No. TESLA-FEL-95-05, DESY, Hamburg, Germany, 1995.

[9] M. Borland, Simple method for particle tracking with coherent synchrotron radiation, Phys. Rev. ST Accel. Beams 4, 070701 (2001).

[10] R. Li, Curvature-induced bunch self-interaction for an energy-chirped bunch in magnetic bends, Phys. Rev. ST Accel. Beams 11, 024401 (2008).

[11] H. H. Braun, R. Corsini, L. Groening, F. Zhou, A. Kabel, T. O. Raubenheimer, R. Li, and T. Limberg, Emittance growth and energy loss due to coherent synchrotron radiation in a bunch compressor, Phys. Rev. ST Accel. Beams 3, 124402 (2000).

[12] S. Dusterer et al., Femtosecond x-ray pulse length characterization at the Linac Coherent Light Source free-electron laser, New J. Phys. 13, 093024 (2011).

[13] S. Di Mitri, D. Castronovo, I. Cudin, and L. Frohlich, Electron slicing for the generation of tunable femtosecond soft x-ray pulses from a free electron laser and slice diagnostics, Phys. Rev. ST Accel. Beams 16, 042801 (2013).

[14] F. Zhou, Slice emittance measurements at the LCLS, (unpublished).

[15] J. Turner et al., FEL over-compression in the LCLS, in Proceedings of the 36th International Free Electron Laser Conference Basel, Switzerland, 2014.

[16] D. Ratner et al., Experimental Demonstration of Soft X-ray Self-Seeded Free Electron Laser, Phys. Rev. Lett. 114, 054801 (2015).

[17] R. Akre et al., Commissioning the linac coherent light source injector, Phys. Rev. ST Accel. Beams 11, 030703 (2008).

[18] Z. Huang et al., Measurements of the linac coherent light source laser heater and its impact on the $\mathrm{x}$-ray free-electron laser performance, Phys. Rev. ST Accel. Beams 13, 020703 (2010).

[19] H. Loos et al., Observation of Coherent Optical Transition Radiation in the LCLS linac, in Proceedings of the 30th International Free Electron Laser Conference, Gyeongju, Korea, 2008, p. 485.

[20] M. Dohlus and T. Limber, Impact of optics on CSR-related emittance growth in bunch compressor chicanes, in Proceedings of the 21st Particle Accelerator Conference, Knoxville, TN, 2005 (IEEE, Piscataway, NJ, 2005). 
[21] G. Stupakov and P. Emma, CSR wake for a short magnet in ultrarelativistic limit, in Proceedings of the 8th European Particle Accelerator Conference, Paris, 2002 (EPS-IGA and CERN, Geneva, 2002), pp. 1479-1481.

[22] M. Borland, ELEGANT, Advanced Photon Source LS-287, 2000.

[23] M. Borland, Simple method of particle tracking with coherent synchrotron radiation, Phys. Rev. ST Accel. Beams 4, 070701 (2001).

[24] P. Emma, Emittance growth mechanics in linac-based Free electron lasers, in Proceedings of the 32nd Free Electron Laser Conference, Malmö, Sweden (Max-lab, Sweden, 2010).
[25] J. Murphy, S. Krinsky, and R. Gluckstern, Longitudinal wakefield for an electron moving on a circular orbit, Part. Accel. 57, 9 (1997).

[26] Y. S. Derbenev, E. L. Saldin, J. Rossbach, and V. D. Shiltsev, Technical Report No. TESLA-FEL-95-5, DESY, 1995.

[27] G. Stupakov, K. L. F. Bane, and I. Zagorodnov, Optical approximation in the theory of geometric impedance, Phys. Rev. ST Accel. Beams 10, 054401 (2007).

[28] K. L. F. Bane, G. Stupakov, and I. Zagorodnov, Impedance calculations of nonaxisymmetric transitions using the optical approximation, Phys. Rev. ST Accel. Beams 10, 074401 (2007). 